\title{
Elaboração e validação de escala diagramática para avaliação da severidade do mofo cinzento em mamoneira
}

\author{
Angelo A.B. Sussel, Edson A. Pozza \& Hilário A. Castro \\ Departamento de Fitopatologia, Universidade Federal de Lavras, 37200-000, Lavras, MG, Brasil
}

Autor para correspondência: Edson Ampélio Pozza, e-mail: eapozza@ufla.br

\section{RESUMO}

Uma escala diagramática foi desenvolvida para padronizar a avaliação do mofo cinzento em cachos de mamoneira, cujo agente etiológico é o fungo Amphobotrys ricini. Foram considerados os limites de severidade mínima e máxima da doença observados no campo, e os níveis intermédiários seguiram incrementos logarítimicos, representando: 3, 5, 8, 12, 25, 50, 76, 86, 94, e 100\% de área necrosada. A validação da escala foi realizada com auxílio de dez avaliadores, e foi dividida em três fases: na primeira fase, os avaliadores analisaram 40 fotografias de cachos com diferentes níveis de severidade, sem o uso da escala diagramática. Na segunda e terceira fases, os mesmos avaliadores estimaram a severidade da doença das mesmas 40 fotografias, apresentadas em ordem diferente, com intervalo entre as avaliações de 7 dias. Por meio de regressão linear, confrontando valores de severidade reais e estimados, e estimativas da primeira e segunda avaliação com uso da escala, analisou-se a acurácia, a precisão, a repetibilidade e a reprodutibilidade das estimativas dos avaliadores. As avaliações com escala diagramática foram precisas e acuradas nas estimativas dos avaliadores, concentrando os erros absolutos dentro dos limites aceitáveis, e proporcionaram alta reprodutibilidade entre as avaliações de diferentes avaliadores. Sendo assim, a escala proposta foi adequada para avaliação da severidade do mofo cinzento em mamona.

Palavras-chave: Amphobotrys ricini, Ricinus communis, quantificação da doença

\begin{abstract}
Elaboration and validation of diagrammatic scale to evaluate gray mold severity in castor bean

A diagrammatic scale was developed to standardize assessment of gray mold in castor bean bunches, caused by Amphobotrys ricini. Both the maximum and minimum limits of disease severity observed in the field were considered, and the intermediate values progressed by logarithmic increments, representing: 3, 5, 8, 12, 25, 50, 76, 86, 94 and 100\% of necrotic area. The scale was validated by 10 raters in three phases: first the raters estimated the severity in 40 photographs without using the scale. In the second and third phases, the same raters estimated the severity in the photographs but with different sequences, with a seven-day interval between assessments. Accuracy, precision, repeatability and reproducibility of estimates were determined by linear regression, relating assessments performed by raters using the scale to actual severity, and relating the results between two assessments using the scale. Assessments with the diagrammatic scale were precise and accurate, the absolute errors were lower than the acceptable limits and the raters exhibited good repetition of estimates. The proposed diagrammatic scale proved to be adequate for severity assessments of gray mold in castor bean.
\end{abstract}

Keywords: Amphobotrys ricini, Ricinus communis, disease quantification.

Dentre as culturas oleaginosas, a mamona (Ricinus communis L.) vem se destacando nos programas energéticos, ligados à agricultura familiar, em todo o território nacional. Entre os fatores associados a perdas, encontram-se as doenças, principalmente o mofo cinzento causado por Amphobotrys ricini (N.F. Buchw.) Hennebert (sin. Botrytis ricini N.F. Buchw.). Em regiões com umidade relativa alta e maior pluviosidade, durante o período reprodutivo da mamoneira, foram descritas perdas superiores a 50\% (Melhorança \& Staut, 2005), em vista da destruição de flores e bagas, deterioração dos grãos e redução do teor de óleo. Apesar de provocar

Parte da Tese de Doutorado do primeiro autor. Universidade Federal de Lavras. Lavras MG. 2008.

*Endereço Atual: Embrapa Cerrados, 73310-970, Planaltina, DF, Brasil. severas perdas e ter sido reportado há quase um século (Godfrey, 1923), até o momento, foram realizados poucos estudos epidemiológicos sobre esta doença. Esses estudos requerem métodos padronizados para avaliar a severidade da doença no campo, que devem ser simples e de rápida utilização em condições diferenciadas. Entre esses métodos destaca-se o uso de escalas diagramáticas (Berger, 1980; Nutter Jr. \& Schultz, 1995).

A utilização de escalas diagramáticas na avaliação de doenças proporciona maior acurácia, precisão e reprodutibilidade nas estimativas de severidade da doença. A acurácia representa o grau de proximidade entre a estimativa e a quantidade real de doença, a precisão refere-se à confiabilidade e/ou repetibilidade nas avaliações da doença e a reprodutibilidade trata da ausência de variação nas estimativas quando a mesma amostra é avaliada por diferentes avaliadores. Até 
o momento, foram utilizadas estimativas diretas da porcentagem do cacho afetado na avaliação da severidade do mofo cinzento (Lima \& Soares, 1990; Milani et al., 2005; Demant et al., 2006; Ueno et al., 2006) que apresentam a inconveniência de serem subjetivas e não seguirem ajustes que levem em conta a acuidade visual para avaliar os diferentes níveis de severidade (Horsfall \& Cowling, 1978; Campbell \& Madden, 1990). Assim, objetivou-se neste trabalho construir e validar uma escala diagramática para avaliar a severidade do mofo cinzento em inflorescências de mamoneira.

Foram coletados cachos de mamona do híbrido Savana e da cultivar IAC Guarani em campo experimental, onde observou-se ampla variação da severidade do mofo cinzento. Os cachos foram fotografados sob fundo azul, com câmera digital. Com auxílio do programa ImageTool ${ }^{\circledR}$ da UTHSCA, determinou-se o percentual de área necrosada de cada cacho fotografado. Baseandose na lei de Weber-Fechner (Horsfall \& Cowling, 1978; Nutter Jr. \& Schultz, 1995) de acuidade visual, e nos níveis máximo e mínimo de severidade observados no campo, foi elaborada uma escala diagramática com dez níveis de severidade, considerando a forma e a distribuição das lesões observadas com maior freqüência. Depois de estabelecidas as porcentagens da doença a serem representadas na escala, reproduziu-se uma imagem do cacho de mamona, de tamanho e formato próximo aos dos cultivares comerciais atualmente utilizados, à qual acrescentou-se desenhos de frutos colonizados pelo fungo, de maneira a retratar os sintomas observados no campo.

Para a validação da escala diagramática, imagens de 40 cachos sintomáticos foram projetadas por meio de projetor tipo "multimídia" para os avaliadores. A severidade da doença foi avaliada por 10 pessoas, alunos do curso de Pós-graduação em Fitopatologia, a maioria sem experiência na quantificação de doenças em mamoneira. Inicialmente, a severidade foi estimada sem auxílio da escala diagramática e após sete dias, com o auxílio da mesma. Visando avaliar a repetibilidade das estimativas com a escala diagramática, uma nova seqüência das mesmas imagens foi organizada e uma segunda estimativa visual com auxílio da escala foi efetuada pelos mesmos avaliadores, sete dias após a primeira avaliação com escala.

A acurácia e a precisão de cada avaliador foram determinadas por meio de regressão linear simples, com a severidade real obtida eletronicamente como variável independente e a severidade estimada como variável dependente. A acurácia das estimativas de cada avaliador e do conjunto de avaliadores foi determinada pelo teste $t$ aplicado ao intercepto da regressão linear $(a)$, para testar a hipótese $\mathrm{H}_{0}: a=0$, e ao coeficiente angular da reta $(b)$, para testar a hipótese $\mathrm{H}_{1}: b=1$, ao nível de $5 \%$ de probabilidade. Valores de intercepto significativamente diferentes de 0 descrevem a presença de desvios constantes, enquanto valores de coeficiente angular da reta significativamente diferentes de 1 indicam a presença de desvios sistemáticos. A precisão das estimativas foi obtida por meio do coeficiente de determinação da regressão $\left(R^{2}\right)$, por erros absolutos (severidade estimada menos severidade real) e pela repetibilidade das estimativas, determinada pela regressão da segunda avaliação em relação à primeira de uma mesma unidade amostral. A reprodutibilidade das estimativas foi determinada por valores de $R^{2}$ obtidos de regressões lineares entre as severidades estimadas entre os diferentes avaliadores combinados em pares (Campbell \& Madden, 1990; Nutter Jr. et al., 1993; Nutter Jr. \& Schultz, 1995). As análises de regressão foram efetuadas com auxílio do software estatístico $\mathrm{SAS}^{\circledR}$.

O valor máximo da severidade, observada no campo, foi de $100 \%$, decorrente de infecção precoce do cacho em período de alta umidade relativa e temperatura em torno de $25^{\circ} \mathrm{C}$, favoráveis à doença (Melhorança \& Staut, 2005). A escala diagramática para quantificar o mofo cinzento da mamoneira foi elaborada com dez níveis de severidade, representados pelos valores de $3,5,8,12,25,50,76,86$, 94, e 100\% de área lesionada (Figura 1).

Apesar de não terem experiência na avaliação de doenças da mamoneira, os avaliadores obtiveram acurácia em suas estimativas, pois apenas $20 \%$ apresentaram valores do intercepto diferente de zero $(\mathrm{P}<0,05)$ para as retas de regressão entre severidade real e estimada, contudo com valor médio de 3,3. Sem a utilização da escala um avaliador superestimou e outro subestimou consistentemente a severidade do mofo cinzento. Com a escala, dois avaliadores em cada avaliação subestimaram a severidade em níveis significativos.

A precisão das estimativas foi considerada alta sem o auxílio da escala, pois os coeficientes de determinação variaram entre 0,89 e 0,97 , com média de 0,93 (Tabela 1). As diferenças (erros absolutos) entre os valores estimados visualmente e os reais obtidos eletronicamente na quantificação da severidade sem o auxílio da escala diagramática variaram entre $-34,59$ e 35,00 (Figura 2). Contudo, todos os avaliadores melhoraram a precisão das estimativas com a utilização da escala, com coeficientes de determinação variando entre 0,92 e 0,98 para a primeira avaliação (Tabela 1), e entre 0,93 e 0,98 para a segunda avaliação, com médias de 0,95 e 0,96 respectivamente, assemelhando-se aos verificados em outros estudos de validação de escalas (Sposito et al., 2004; Halfeld-Vieira \& Nechet, 2006).

Houve redução dos valores dos erros absolutos das estimativas com o auxílio da escala diagramática, quando comparados com os valores dos erros absolutos das estimativas obtidas sem a utilização da escala. Com a utilização da escala diagramática, os erros absolutos não apresentarampadrãotendenciosoquerefleteasuperestimação ou subestimação da severidade, e os mesmos variaram na primeira e segunda avaliações utilizando a escala entre $-33,00$ e 30,00 , e $-25,00$ e 20,00 , respectivamente (Figura 2). Para valores de severidade inferiores a $20 \%$ 

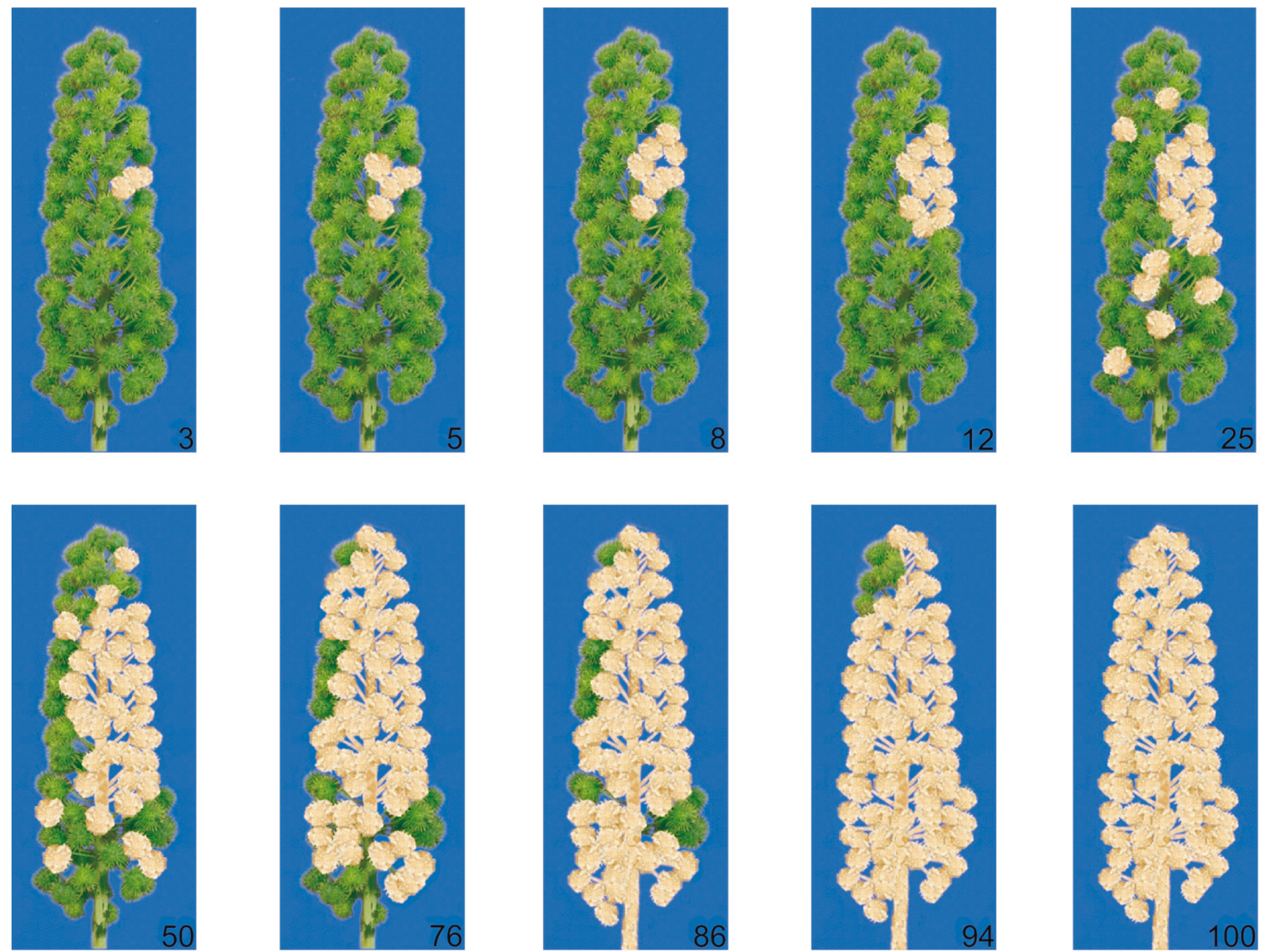

FIGURA 1 - Escala diagramática de severidade do mofo cinzento causado por Amphobotris ricini, em cachos de mamona.
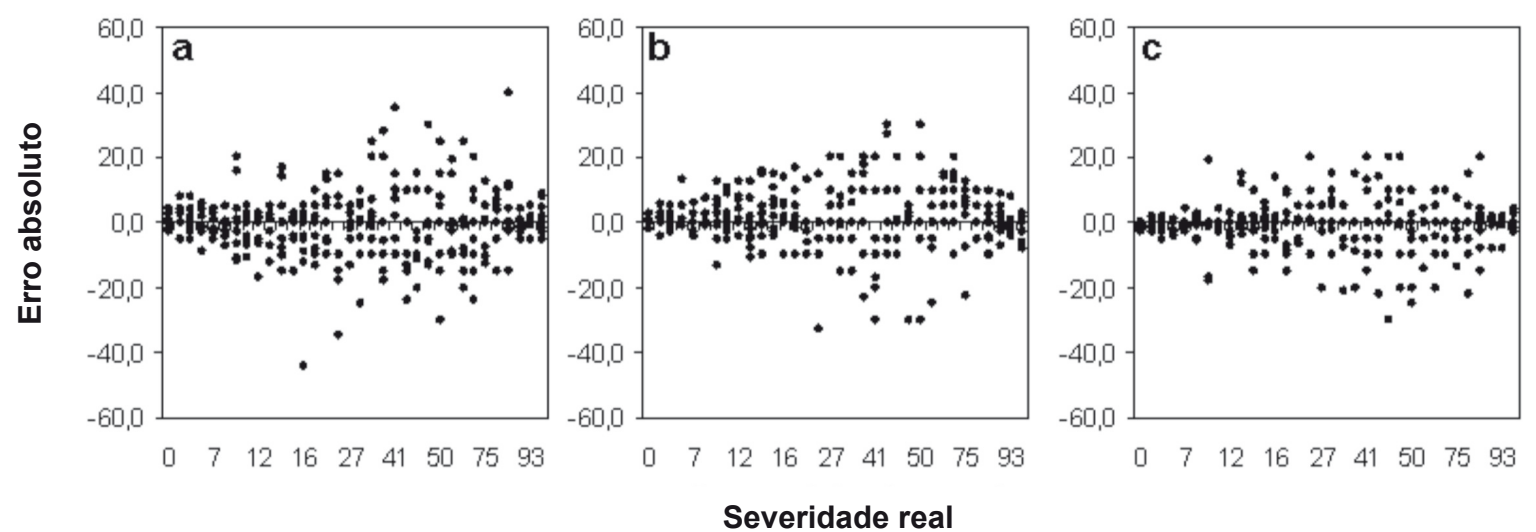

FIGURA 2 - Erros absolutos (diferenças entre severidades estimada e real) de todos os avaliadores, em avaliação sem uso da escala diagramática (A), e com o uso da escala diagramática (B-C). Pontos representam as 40 estimativas de cada avaliador. 
e superiores a $80 \%$, os valores dos erros absolutos foram menores que nos outros níveis de severidade. A maioria dos erros absolutos dos avaliadores que utilizaram a escala foi inferior a $10 \%$. Segundo os critérios adotados em estudos de avaliação de escalas diagramáticas, os erros absolutos obtidos na validação são aceitáveis (Amorim et al., 1993; Nutter Jr. \& Schultz, 1995; Sposito et al., 2004; Nascimento et al., 2005; Michereff et al., 2000, 2006; Barbosa et al., 2006), porém, podem ser minimizados com o treinamento dos avaliadores (Nutter Jr. \& Schultz, 1995).

A princípio, observou-se tendência de subestimar os valores de severidade, visto que os valores de intercepto na primeira avaliação foram todos negativos, porém o treinamento com a escala pode minimizar esta condição conforme observado na segunda avaliação, quando dois avaliadores deixaram de subestimar a doença. Michereff et al. (2000) também observaram que o treinamento dos avaliadores contribuiu para melhorar os índices de precisão e acurácia na avaliação da severidade da queima das folhas de inhame.

Os avaliadores relataram sentir maior dificuldade de estimar a severidade de cachos com baixa densidade de frutos, diferentes dos cachos utilizados na composição da escala. Tal comportamento já foi relatado por Kranz (1988), onde discute que a qualidade da estimativa da doença pode ser influenciada pela complexidade amostral como o tamanho, a forma, a cor e o número de lesões da unidade amostral.

Os avaliadores apresentaram boa repetibilidade nas estimativas, o que pode ser observado nos resultados da regressão entre a primeira e a segunda avaliação. A média do coeficiente de determinação de todos avaliadores foi de $96 \%$, apenas dois avaliadores apresentaram valor de coeficiente angular da reta significativamente diferente de $1(\mathrm{P} \leq 0,01)$. Apenas um avaliador apresentou valor de intercepto diferente de zero $(\mathrm{P} \leq 0,01)$ (Tabela 2$)$, isto ocorreu devido o avaliador subestimar a severidade no primeiro uso da escala e superestimar a severidade no segundo uso da escala, contudo os valores do coeficiente angular da reta são significativamente iguais a um em ambos casos (Tabela 1).

A reprodutibilidade das estimativas entre os avaliadores também foi utilizada como indicativo de análise da precisão da escala. Segundo Nutter Jr. et al. (1993), diferentes avaliadores utilizando uma mesma escala e avaliando o mesmo material, devem estimar os mesmos valores de severidade, cuja significância é verificada por meio de regressões lineares entre as severidades estimadas pelos avaliadores, aos pares. Sem a utilização da escala diagramática, o coeficiente de determinação $\left(\mathrm{R}^{2}\right)$ das regressões das estimativas entre os pares de avaliadores variou de 82 a 97\%, com média de 91\% (Tabela 3). Com a utilização da escala diagramática, os valores de $\mathrm{R}^{2}$ variaram de 87 a 98\%, com média de 94\%, assemelhandose ao constatado na validação de escalas diagramáticas para outros patossistemas (Nascimento et al., 2005; Michereff et al., 2006). Sendo assim a reprodutibilidade das avaliações foi garantida com o uso da escala diagramática.

A padronização da avaliação da severidade do mofo cinzento da mamona é desejável e necessária, pois a uniformização da metodologia de avaliação permitirá comparar resultados obtidos em experimentos em diferentes locais, variedades, métodos de controle entre outros. A

TABELA 1 - Intercepto $(a)$, coeficiente angular da reta $(b)$ e coeficiente de determinação $\left(R^{2}\right)$ das equações de regressão linear calculadas entre severidade real e severidade estimada do mofo cinzento, efetuadas por avaliadores com e sem o auxilio de escala diagramática

\begin{tabular}{|c|c|c|c|c|c|c|c|c|c|}
\hline \multirow[t]{3}{*}{ Avaliador } & \multirow{2}{*}{\multicolumn{3}{|c|}{ Sem Escala }} & \multicolumn{6}{|c|}{ Com Escala } \\
\hline & & & & \multicolumn{3}{|c|}{$1^{a}$ Avaliação } & \multicolumn{3}{|c|}{$2^{\mathrm{a}}$ Avaliação } \\
\hline & $a$ & $b$ & $R^{2}$ & $a$ & $b$ & $R^{2}$ & $a$ & $b$ & $R^{2}$ \\
\hline A & $6,33 * 1$ & 0,96 & 0,92 & $-2,61$ & 1,05 & 0,97 & $-3,58^{*}$ & 1,06 & 0,96 \\
\hline B & 1,83 & 0,96 & 0,97 & $-1,46$ & 1,00 & 0,97 & $-0,09$ & 0,95 & 0,96 \\
\hline $\mathrm{C}$ & 1,03 & 1,01 & 0,95 & $-1,97$ & 1,01 & 0,96 & 0,33 & 0,97 & 0,95 \\
\hline $\mathrm{D}$ & $-5,34$ & 1,07 & 0,91 & $-2,50$ & 1,08 & 0,94 & $-1,25$ & $1,12 *$ & 0,94 \\
\hline $\mathrm{E}$ & 1,47 & 1,11 & 0,92 & $-3,46$ & $1,11^{*}$ & 0,96 & $-3,43$ & 1,10 & 0,95 \\
\hline $\mathrm{F}$ & $-6,69 *$ & 1,09 & 0,89 & $-1,93$ & 1,01 & 0,92 & $-2,50$ & $1,13^{*}$ & 0,93 \\
\hline G & $-1,47$ & 1,09 & 0,92 & $-4,78^{*}$ & 1,08 & 0,97 & $-3,57$ & 1,09 & 0,93 \\
\hline $\mathrm{H}$ & $-4,64$ & 0,99 & 0,90 & $-4,58 *$ & 1,09 & 0,97 & $-4,94 *$ & 1,03 & 0,96 \\
\hline I & 3,86 & 0,96 & 0,94 & $-2,97$ & 1,03 & 0,92 & 0,45 & 1,00 & 0,94 \\
\hline $\mathrm{J}$ & $-0,31$ & 1,02 & 0,97 & $-2,99$ & 1,05 & 0,98 & $-1,98$ & 1,05 & 0,98 \\
\hline Média & & & 0,93 & & & 0,96 & & & 0,95 \\
\hline
\end{tabular}

*1 indica que a hipótese de nulidade $(a=0$ ou $b=1)$ foi rejeitada pelo teste $t(\mathrm{P}=0,05)$. 
TABELA 2 - Intercepto $(a)$, coeficiente angular da reta $(b)$ e coeficiente de determinação $\left(R^{2}\right)$ das equações de regressão linear relacionando a segunda à primeira estimativa de mofo cinzento, por um mesmo avaliador, com auxílio da escala diagramática

\begin{tabular}{lccc}
\hline \hline Avaliador & $\boldsymbol{a}$ & $\boldsymbol{b}$ & $\boldsymbol{R}^{\mathbf{2}}$ \\
\hline A & 1,44 & 0,96 & 0,98 \\
B & $-1,08$ & 1,04 & 0,99 \\
C & $-0,71$ & 0,99 & 0,93 \\
D & $-0,38$ & 0,94 & 0,95 \\
E & 0,68 & 0,99 & 0,97 \\
F & 1,68 & $0,86^{*}$ & 0,91 \\
G & 0,60 & 0,93 & 0,94 \\
H & 1,52 & 1,03 & 0,96 \\
I & $-3,67 * 1$ & $1,05^{*}$ & 0,99 \\
J & $-0,79$ & 0,99 & 0,99 \\
\hline Média & & & 0,96
\end{tabular}

*1indica que a hipótese de nulidade $(a=0$ ou $b=1)$ foi rejeitada pelo teste $t(\mathrm{P}=0,01)$.
TABELA 3 - Coeficiente de determinação $\left(R^{2}\right)$ de equações de regressão linear relacionando as estimativas do mofo cinzento entre avaliadores com e sem o auxílio da escala diagramática (primeira e segunda avaliações)

\begin{tabular}{|c|c|c|c|c|c|c|c|c|}
\hline \multirow[b]{2}{*}{ AVALIADOR } & \multicolumn{6}{|c|}{ Sem escala } & \multirow[b]{2}{*}{$\mathbf{H}$} & \multirow[b]{2}{*}{ I } \\
\hline & B & $\mathrm{C}$ & D & $\mathbf{E}$ & $\mathbf{F}$ & G & & \\
\hline A & 0,92 & 0,95 & 0,83 & 0,89 & 0,87 & 0,90 & 0,87 & 0,92 \\
\hline B & & 0,96 & 0,92 & 0,95 & 0,89 & 0,92 & 0,95 & 0,95 \\
\hline $\mathrm{C}$ & & & 0,88 & 0,94 & 0,87 & 0,88 & 0,91 & 0,96 \\
\hline $\mathrm{D}$ & & & & 0,91 & 0,82 & 0,83 & 0,88 & 0,88 \\
\hline $\mathrm{E}$ & & & & & 0,91 & 0,90 & 0,87 & 0,93 \\
\hline $\mathrm{F}$ & & & & & & 0,85 & 0,85 & 0,91 \\
\hline G & & & & & & & 0,84 & 0,91 \\
\hline $\mathrm{H}$ & & & & & & & & 0,91 \\
\hline \multicolumn{9}{|l|}{ I } \\
\hline \multicolumn{9}{|c|}{ Com escala - $1^{\text {a }}$ Avaliação } \\
\hline & B & $\mathrm{C}$ & D & $\mathbf{E}$ & $\mathbf{F}$ & G & $\mathbf{H}$ & I \\
\hline A & 0,93 & 0,91 & 0,94 & 0,96 & 0,88 & 0,97 & 0,97 & 0,90 \\
\hline B & & 0,96 & 0,93 & 0,94 & 0,93 & 0,97 & 0,96 & 0,91 \\
\hline $\mathrm{C}$ & & & 0,90 & 0,92 & 0,91 & 0,93 & 0,94 & 0,88 \\
\hline $\mathrm{D}$ & & & & 0,95 & 0,93 & 0,94 & 0,91 & 0,91 \\
\hline E & & & & & 0,91 & 0,96 & 0,96 & 0,91 \\
\hline $\mathrm{F}$ & & & & & & 0,92 & 0,91 & 0,93 \\
\hline G & & & & & & & 0,96 & 0,93 \\
\hline $\mathrm{H}$ & & & & & & & & 0,91 \\
\hline \multicolumn{9}{|l|}{ I } \\
\hline \multicolumn{9}{|c|}{ Com Escala - 2a Avaliação } \\
\hline & B & $\mathrm{C}$ & D & $\mathbf{E}$ & $\mathbf{F}$ & G & $\mathbf{H}$ & I \\
\hline A & 0,96 & 0,95 & 0,95 & 0,95 & 0,95 & 0,94 & 0,97 & 0,93 \\
\hline B & & 0,94 & 0,93 & 0,94 & 0,93 & 0,90 & 0,97 & 0,95 \\
\hline $\mathrm{C}$ & & & 0,92 & 0,94 & 0,92 & 0,93 & 0,95 & 0,94 \\
\hline $\mathrm{D}$ & & & & 0,92 & 0,91 & 0,93 & 0,93 & 0,95 \\
\hline $\mathrm{E}$ & & & & & 0,93 & 0,93 & 0,93 & 0,93 \\
\hline $\mathrm{F}$ & & & & & & 0,87 & 0,94 & 0,93 \\
\hline G & & & & & & & 0,91 & 0,89 \\
\hline $\mathrm{H}$ & & & & & & & & 0,94 \\
\hline I & & & & & & & & \\
\hline
\end{tabular}

\section{REFERÊNCIAS BIBLIOGRÁFICAS}

de Minas Gerais - FAPEMIG pela concessão de bolsa de estudo ao primeiro autor e auxílio financeiro para realização do trabalho (Proc. APQ-3525-3.09/07). Aos pós-graduandos do Departamento de Fitopatologia e Departamento de Agricultura da UFLA pelo auxílio na validação da escala diagramática.

\section{AGRADECIMENTOS}


Angelotti F, Scarpin CR, Tessmann D, Vida JB, Oliveira RR, Canteri MG (2008) Diagramatic scale for assessment of grapevine rust. Tropical Plant Pathology 33:439-443.

Barbosa MAG, Michereff SJ, Mora-Aguilera G (2006) Elaboração e validação de escala diagramática para avaliação da severidade da ferrugem branca do crisântemo. Summa Phytopathologica 32:5762.

Berger RD (1980) Measuring disease intensity. In: Teng PS, Krupa SV (Eds.) Crop loss assessment. Saint Paul MN. University Minessota. pp. 28-31.

Campbell CL, Madden LV (1990) Introduction to plant disease epidemiology. New York NY. Wiley Interscience.

Demant CAR, Furtado EL, Zanotto MD, Chagas AA (2006) Controle de mofo cinzento com o uso de Trichoderma. In: 2. Congresso Brasileiro de Mamona. Salvador BA. Embrapa Algodão. CD-ROM.

Godfrey GH (1923) Gray mold of castor bean. Journal of Agricultural Research 22:679-715.

Halfeld-Vieira BA, Nechet KL (2006) Elaboração e validação de escala diagramática para avaliação da mancha-de-cercospora em melancia. Fitopatologia Brasileira 31:46-50.

Horsfall JG, Cowling EB (1978) Pathometry: the measurement of plant disease. In: Horsfall JG, Cowling EB (Eds.) Plant Disease: an advanced treatise-how disease develops in populations. New York NY. Academic Press.

Kranz J (1988) Measuring plant disease. In: Kranz J, Rotem J (Eds.) Experimental techniques in plant disease epidemiology. Heidelberg. Springer-Verlag.

Lima EF, Soares JJ (1990) Resistência de cultivares de mamoneira ao mofo cinzento causado por Botrytis ricini. Fitopatologia Brasileira 15:96-97.
Melhorança AL, Staut TA (2005) Indicações técnicas para a cultura da mamona em Mato Grosso do Sul. Dourados. Embrapa Agropecuária Oeste.

Michereff SJ, Andrade DEGT, Noronha MA (2006) Elaboração e validação de escala diagramática para avaliação de severidade do carvão da folha do caupi. Summa Phytopathologica 32:51-56.

Michereff SJ, Maffia LA, Noronha MA(2000) Escala diagramática para avaliação da severidade da queima das folhas do inhame. Fitopatologia Brasileira 25:612-619.

Milani M, Nobrega MBM, Suassuna ND, Coutinho WM (2005) Resistência da mamoneira (Ricinus communis L.) ao mofo cinzento causado por Amphobotrys ricini. Campina Grande PB. Embrapa Algodão. Documentos 137.

Nascimento ARP, Michereff SJ, Mariano RLR, Gomes AMA, (2005) Elaboração de escala diagramática para o cancro bacteriano da videira. Summa Phytopathologica 31:59-64.

Nutter Júnior FW, Gleason ML, Jenco JH, Christians NC (1993) Assessing the accuracy, intra-rater repeatability, and inter-rater reliability of disease assessment systems. Phytopathology 83:806812.

Nutter Júnior FW, Schultz PM (1995) Improving the accuracy and precision of disease assessments: selection of methods and use of computer-aided training programs. Canadian Journal of Plant Pathology 17:174-184.

Sposito MB, Amorim L, Belasque Junior J, Bassanezi RB, Aquino R (2004) Elaboração e validação de escala diagramática para avaliação da severidade da mancha preta em frutos cítricos. Fitopatologia Brasileira 29:81-85.

Ueno B, Hellwig TC, Nickel G, Silva SDA (2006) Resistência ao mofo cinzento em 15 genótipos de mamoneira cultivadas na região de pelotas, RS, safra 2004/2005 In: 2 Congresso Brasileiro de Mamona. Campina Grande. Embrapa Algodão. CD-ROM.

Recebido 18 Julho 2008 - Aceito 08 Junho 2009 - TPP 8078

Editor Associado: Nilceu R.X. Nazareno 